\title{
3. VARIABLE STARS IN THE ORION NEBULA
}

\section{Frequency and Distribution}

\section{Rosino}

Irregular variable stars in the Trapezium region were first announced by Bond ( $\mathbf{r}$ ) about a century ago. After the introduction of photographic techniques for the search, and the use of infra-red emulsions, the number of variables steadily increased and now more than 500 variable stars associated with the Orion Nebula and its immediate surroundings are known.

The survey, however, is by no means complete for the following reasons:

(a) The presence of a strong nebular continuum, which reduces the limiting magnitude near the Trapezium (Huyghenian Region), where many faint stars are clustered;

(b) The intrinsic faintness of most of the nebular variable stars;

(c) The relatively high frequency of that type of variable which, like the flare stars, normally is at minimum and only rarely undergoes light changes of very short duration.

These factors and the use of telescopes of different size and characteristics in the searches, are responsible for selection effects in the distribution and completeness of the surveys. It is apparent, however, that the distribution of the known variables in Orion follows that of the stars associated with the groups of diffuse nebulae NGC 1976, 1982, 1977 and 1999. The association of the variables with the bright and dark nebulosities is striking. The frequency is particularly high in the very well searched area around the Trapezium (NGC 1976 and I982) where at least 70 per cent of the stars up to $17.5 \mathrm{pg}$ appear to be variable. Observers actually have difficulty in finding comparison stars of constant brightness in this area. Variable stars are found in great numbers near the nebula NGC 1977, north of the Trapezium (82 variables, 30 per cent of the stars) and in the area of NGC 1999 (60 variables, 25 per cent of the stars), as well as in the region of the Horsehead Nebula (IC 434, NGC 2024) and $\lambda$ Orionis $(2,3,4,5)$. All of these variables undoubtedly belong to the Orion Complex and have, from the point of view of light variability, the same features. The frequency per unit area obviously depends upon the total number of stars involved in the nebulosity and the absorption inside the Nebula. In heavily obscured regions, variables are frequently found only in the fringes, where the absorption becomes smaller and the stars imbedded in the nebulosity can be seen. The number, however, drops suddenly to zero when the density of the nebulous matter vanishes and the rich background of stars reappears.

\section{Light Curves, Amplitudes, Luminosities}

Although the light curves of the variable stars in the Orion Complex have obvious characteristics in common, it is difficult to find a suitable classification on account of the extreme irregularity of the brightness fluctuations. An analysis of the photometric material now available gives support to the following classification due to Parenago (6):

I. Brightness more frequently near minimum, with irregular deep minima (AI, AH, AZ, MO Ori). In some of the variables the occurrence of minima at fairly regular intervals may suggest eclipses. However, after some cycles have elapsed, the apparent regularity disappears and the light curves again become erratic.

II. Irregular variations of moderate amplitude around an intermediate magnitude (NP, NQ, KN Ori). The fluctuations sometimes are very small and for months, stars of this class may appear nearly constant.

III. Brightness usually near minimum with sudden sharp maxima (XY, $\mathrm{V}_{3} 88, \mathrm{IZ}, \mathrm{MY}$ Ori).

IV. Erratic fluctuations without preference for any level between maximum and minimum (XZ, OT, WX Ori). 
In the writer's opinion class III is the best defined. Variables of types I, II and IV pass at times from one class to another. Stars of class I and IV occasionally display considerable activity with rapid irregular oscillations of large amplitude. Sudden changes of brightness (not having the character of flares) have been observed in some of these stars (HS, YY, B637, SU Ori) to occur in the space of 1 or 2 hours.

Statistical analysis of the light curves would be of great importance and has been tried in the past, but without significant results. The major difficulty is that of obtaining good light curves over a period of several years because of the lack of extended and homogeneous series of observations and the poor quality of the estimated magnitudes. Apparently there is no relation between the form of the light curve and the absolute magnitude and spectrum. Also the presence or absence of emission lines does not seem to influence the characteristics of the light curves. However, Götz (5) has recently found that the intensity of $\mathrm{H} \alpha$ is smaller when rapid variations occur. This and other relations between physical parameters and brightness fluctuations should be the main object of future investigations. The explorative phase in the study of Orion variables is nearly complete, at least in some areas and to a certain limiting magnitude. What is now required is an accurate survey, over a period of years, of individual variables of the most representative types, both spectroscopically and photometrically, with the purpose of correlating the observed peculiarities of the light curves with changes of colour or spectrum.

The mean amplitude of the nebular variables in Orion is $A=I^{m} \cdot 60$. However, there are large deviations from the mean value. Variables of the classes I and IV like MO, OT, WX, AZ Ori, etc. sometimes have amplitudes from $2^{m} \cdot 5$ to $4^{m} \cdot 5$. To the contrary, variables of class II, like NP, $\mathrm{V}_{3} 87, \mathrm{~V}_{355}, \mathrm{~V}_{35} 8$ Ori have fluctuations of smaller amplitude, normally under $\mathrm{I}^{m}$. No large differences exist between the mean amplitude of the nebular variables in Orion and that of nebular variables in NGC $2264\left(A=I_{1}{ }^{m} 5^{\circ}\right)$, Taurus $\left(A=I_{1}^{m} \cdot 40\right)$ and NGC 7023 $\left(A={ }_{1}{ }^{m} \cdot 70\right)$.

The frequency distribution of median photographic magnitudes for 424 variables in a circular area of $5^{\circ}$ diameter centred in the Trapezium, has been examined. Selection effects in the discovery are clearly shown for stars fainter than magnitude $\mathbf{I} 7$, since the limiting magnitude of the telescopes hitherto used in surveys of the Orion Nebula rarely is fainter than $18^{m}$. Considering only variables brighter than $\mathrm{I} 7^{m}$ and assuming for the Trapezium a distance modulus of $+8 \cdot 5$, it clearly appears that the median absolute magnitudes of the variables in Orion are concentrated between +4 and +9 . Variables of class III, if flare stars are included, are in general the faintest. Only a small number of variables are brighter than +4 , and it should be observed that the variability of some of them is doubtful. The inferior limit of the luminosity function is not defined, since very little is known about variables fainter than apparent magnitude $\mathbf{I} 8$.

\section{Spectra}

In a coarse classification the variables of the Orion Nebula can be subdivided, from the spectroscopic point of view, in three groups:

(a) Early-type stars with emission lines ( $\mathrm{T}, \mathrm{V}_{3} 8 \mathrm{o}, \mathrm{HK}$ Ori, etc.)

(b) T Tauri and T Tauri-like stars (GX, HI, VZ, WX, SU, SW, IX, YY, LX Ori, etc.)

(c) Non-emission variables (MO, MP, MQ, MS, AH Ori, etc.)

Practically all of the stars of the Orion Complex having emission lines are found to be variable when they are sufficiently observed.

It is known from Joy, Herbig (6), and Haro that many $T$ Tauri stars of the Orion Nebula (SU, NS, KP, AU, VY, XX, YY Ori, etc.) show an excess of ultra-violet radiation and a veiling by continuous radiation of the absorption spectrum, whose physical nature remains unknown. In some of these stars Walker (7) has recently found redward-displaced hydrogen absorption lines by +150 to $300 \mathrm{~km} / \mathrm{sec}$ (he has called this the inverse P Cyg effect) which are likely to 
indicate the presence of infalling material. The interpretation of the facts, however, is rather intriguing, if we consider that in many $\mathrm{T}$ Tauri stars there are also traces of mass ejection indicated by the presence of shortward absorption components of $\mathrm{H}$ and $\mathrm{Ca}$ II emission lines (8). Analysis of the light curves in order to see whether there are differences between emission and non-emission variables and between UV-excess stars with or without the inverse P Cygni effect have been recently made at Asiago, on special series of plates taken during the last winter. A preliminary study of these plates does not show significant differences.

Variables of advanced spectral type in the Orion Nebula fall generally (but not always) to the right and above the main sequence, as in other young nebular clusters, although with much larger dispersion. This fact is currently interpreted as an indication that gravitational contraction is still in process on these stars. Variability may be an effect of internal perturbation induced by contraction while the star is evolving towards the main sequence. But this is also a point that should be clarified by further studies.

\section{Flare stars}

Of extremely high interest among the variables of the Orion Nebula are the 'flare stars' or 'flash stars' or 'rapid variables', first discovered by Morgan and Haro in 1953. The flare stars stay normally at minimum, where they present only small fluctuations of brightness. When an outburst occurs there is a sudden and extremely rapid increase of luminosity followed by a slower return to minimum. The time to go from minimum to maximum and again to minimum varies from less than 40 minutes to 5 hours (9). The amplitude ranges from $0^{m} \cdot 5$ to $4^{m}$, but at times flareups of smaller amplitude also occur. The spectra at minimum are from $\mathrm{dK} 2$ to dMo (ro). Emission lines are mostly, but not always, faint or absent. Many of these objects during their quiescent stage are practically identical, from the spectroscopic point of view, with normal late-type dwarf stars. During an outburst the emission lines are strengthened and a strong continuous emission is noticed in the violet and ultraviolet. An analysis of the material fully supports the conclusions formulated by Haro some years ago:

(a) The distribution and frequency of flare stars in Orion are closely correlated with the distribution and frequency of nebular variables.

(b) The uncorrected absolute magnitudes of flare stars fall between +6 and + I or less, the fainter limit being indefinite.

(c) The luminosity variations in flares are mainly due to $\mathrm{H} \alpha$ and blue-ultraviolet emissions. The change in the infra-red is small or absent. In fact no infra-red flares have been discovered thus far.

(d) The frequency of flares in a given star is not high. The mean spacing between two successive flares can be estimated as of the order of several days or more.

Flares in the Orion Nebula have a relatively longer duration than in other associations, like Taurus or Monoceros (NGC 2264). Haro and Chavira have tried to correlate the total time of an outburst with the spectral type at minimum, concluding that there is a definite relation between spectrum and rate of variation, in which also the UV Ceti variables might be included. According to this relation the rapidity of an outburst increases with increasing spectral type, which is $\mathrm{K}_{5}-\mathrm{K}_{6}$ in Orion and $\mathrm{M}_{2}-\mathrm{M}_{5}$ in Taurus. Further researches of Haro, who has recently discovered large numbers of flare stars in Orion, Pleiades, Praesepe and Coma, indicate that there may be a relation between the frequency of outbursts in a flare star and the age of the cluster, the frequency increasing with age.

Simultaneous photographic and radio surveys of the Orion Complex have been made in the last winter ( $1963-64)$ by the Observatories of Asiago and Tonantzintla and the Lincoln Laboratories of Bedford, Massachusetts, in order to determine whether optical outbursts might be associated with radio bursts. Preliminary analysis of the material, however, does not show any significant coincidences. 


\section{Peculiar variable objects}

Peculiar variable objects whose nature is somewhat difficult to define are found in the Orion Complex. These objects can be classified in the following classes:

(a) Infra-red variables. These are visible only on photographs in the deep red or infra-red. Very little is known of their characteristics. The light curves do not differ substantially from those of other variables. Strong selective absorption and low temperature may be responsible of their high infra-red colour indices.

(b) Infra-red variable stars embedded in minute nebulosities. The difference from the preceding objects is that in the infra-red, small nebulosities become visible around the stars. Sometimes the infra-red stars appear slightly nebulous (OW Ori).

(c) Herbig-Haro Objects. The spectra of these well known Objects have the following lines in emission: $\mathrm{H} \alpha$ and hydrogen lines; [OI] $\lambda \lambda 6300,6363$; [OII] $\lambda \lambda 3727-29$; strong [SII] $\lambda \lambda 6717-31$ and $\lambda \lambda$ 4068-4076. Accompanying this nebular spectrum is a weak continuum of advanced spectral type, with $\mathrm{Ca}$ II and $\mathrm{Fe}$ II in emission, veiled by the nebular spectrum. Probably related to the Herbig-Haro Objects are some roundish minute nebulosities with $\mathrm{H} \alpha$ and [SII] $\lambda \lambda 67 \mathrm{I}_{7}-3 \mathrm{I}$ in emission. The Herbig-Haro Objects are likely not to be stars, but semistellar nebulous knots. They are included here because some of them have shown variability.

\section{Conclusions}

Variable stars in the Orion Complex offer problems of the highest interest for a better understanding of the first stages of stellar evolution and the correlation between stars and surrounding nebular medium. It is likely that an evolutionary sequence in the sense of decreasing intensity of the emission features may be established, as it was proposed by Haro (9), for variable stars ranging from the Herbig-Haro Objects and UV emission variables to stars with weak emission, through non-emission variables, to flare stars. There is, however, a point that should be stressed here: despite the fact that these stars have different physical characteristics, a large spread in luminosities and spectra, with the exception of the flare variables they all have similar light curves, with the same, small or large, irregular variations, and this similarity is apparently independent of spectral type and intrinsic brightness. This fact must have a physical significance; it should be better analysed in future investigations, as it is of great importance for a complete understanding of the phenomena of variability in very young stars.

In conclusion, further work on the Orion variables should be directed to the following points:

(a) A complete survey for new variables in all of the Orion Complex, extended down to magnitude $2 \mathrm{I}$ in the Trapezium region. An extension to magnitude $2 \mathrm{I}$ will give a better knowledge of the luminosity function, which is now indeterminate in the faint limit.

(b) An extensive search for flare stars, with observations made from different longitudes in order to discover the frequency of the outbursts.

(c) Location of the Orion variables in a revised $\mathrm{H}-\mathrm{R}$ diagram of the region. Good sequences of comparison stars in $U, B, V$ and infra-red should be established down to the faintest magnitudes.

(d) A photometric survey in several colours $(U, B, V$, and $I)$ for a selected number of the most representative variables, and simultaneous observations of their spectra.

(e) A photo-electric study of the rapid fluctuations of nebular variables and flare stars at minimum.

\section{REFERENCES}

x. Bond, G. P. Harv. Annals, 5, 124, 1867.

2. Rosino, L. Bologna Pubbl., 5, 1, 1946; Contr. Obs. Asiago no. 69, 1956; no. 125, 1963. 
3. Maffei, P. Contr. Obs. Asiago no. 136, 1963; no. 140, 1963.

4. Rosino, L. In press.

5. Götz, W. Veröff. Sternw. Sonneberg, 4, 245, 1958.

6. Herbig, G. Adv. Astr. and Astrophys., 1, 47, 1962.

7. Walker, M. C. R. Acad. Sci. Paris, 253, 383,$1961 ; R$. Obs. Bull. no. 82, 1964.

8. Herbig, G. Adv. Astr. and Astrophys., I, $87,1962$.

9. Haro, G. Astr. F., 65, 490, I960; Symposium on Stellar Evolution, La Plata, p. 37, I962.

ro. Herbig, G. Astrophys. F., I35, 736, 1962.

II. Joy, A. H. in Stellar Atmospheres, p. 66r, I96r.

\section{FLARE STARS IN THE ORION NEBULA}

\section{G. Haro}

A large number of plates have been obtained over the past ro years at the Tonantzintla Observatory in a search for flare stars; they are centred on the Trapezium and cover an area of 20 sq. degrees. Rosino and his colleagues have also searched for flare stars in the same field. Altogether, I2I stars with flare activity have now been discovered in this area. Their distribution on the sky is not random, but follows the general distribution of the $T$ Tauri-like stars in the region.

Our colour-magnitude diagram of $V$ vs. $B-V$ of the flare stars in Orion shows the following interesting feature: the flare stars appear abruptly at about $V \cong 13.5$, with a colour of about $+\mathrm{I} \cdot 0$ magnitude. Practically all the brightest flare stars lie above the main sequence; the horizontal departure from the main sequence is from several tenths of a magnitude to the blue of the main sequence up to nearly 4 magnitudes towards the red. To still fainter magnitudes, beyond $V=16.0$ about half of the flare stars lie on or above the main sequence, while the other half lie below.

The earliest spectral type of a flare star in the Orion Nebula is between Ko and K2. In NGC 2264, we found that the earliest was about $\mathrm{K}_{\mathrm{I}}$. Of course, there are many other flare stars in both aggregates as late as $\mathrm{M}_{2}$, or even later. As we pass from these young aggregates to older groups, we notice immediately that the spectral types of the brightest flare stars (at minimum light) found in these older organizations become later. For instance, in the Pleiades the brightest known flare stars are $\mathrm{K}_{5}$, while in Coma, Praesepe, the Hyades, and the solar neighbourhood all are of type M. On this evidence, it seems probable that the earliest type possible for a flare star is about $\mathrm{KI}$, which should mark in the $\mathrm{H}-\mathrm{R}$ diagram a left-hand boundary for the appearance of this kind of object. Perhaps there is also a right-hand boundary defined by stars of very low mass $(\leqslant 0.07 \odot)$ which, due to internal degeneracy, cannot remain on the main sequence.

In the Orion Nebula, there is a remarkable spread in the spectral types of the flare stars as well as in their vertical scatter both above and below the main sequence. This might be caused by a difference in the ages and in the evolutionary states of the flare stars in this aggregate. The location of such stars well below the main sequence-if real-constitutes an apparent discrepancy between observation and the conventional theory of gravitational contraction.

A good part of our observational results gives strong empirical support to Hayashi-type contraction, and conversely, Hayashi's theory seems to explain and clarify some of our observations. For example, if one draws in a theoretical H-R diagram that curve which is the locus of the intersection of the purely convective tracks with the purely radiative ones for a range of 